\title{
Evaluation of risk factors for insulin resistance: a cross sectional study among employees at a private university in Lebanon
}

Myriam Fahed ${ }^{1}$, Maya G. Abou Jaoudeh', Samar Merhi ${ }^{1}$, Jocelyne Matar Bou Mosleh${ }^{1}$, Rachelle Ghadieh ${ }^{1,2}$, Sibelle Al Hayek ${ }^{1,3}$ and Jessy E. El Hayek Fares ${ }^{1 *}$

\begin{abstract}
Background: Worldwide, the prevalence of insulin resistance ranges from 15.5 to $46.5 \%$, among adults. Lebanon reported one of the highest rates reaching $44.6 \%$. The literature suggests an association between dairy product consumption and insulin resistance, however results are inconclusive. To our knowledge, no study examined this association in the Middle Eastern Region. The aim of the study was to investigate the prevalence of insulin resistance among a sample of Lebanese adults, to identify its risk factors depending on gender, and to evaluate the association between insulin resistance and dairy products consumption.

Methods: A cross-sectional study was conducted among employees at Notre Dame University - Louaize. Four questionnaires were administered including a background and International Physical Activity Questionnaire short form questionnaires, food frequency questionnaire and a $24 \mathrm{~h}$ recall. Bioelectric Impedance Analysis (BIA) was used to measure percent body fat (PBF). Homeostatic Model Assessment for Insulin Resistance (HOMA-IR) was used to quantify insulin resistance. A person with $\mathrm{HOMA-IR} \geq 2.5$ was considered as insulin resistant. Statistical analyses were performed using the Statistical Package for Social Sciences version 23 for Windows. $P<0.05$ was considered statistically significant.

Results: Out of 286 study participants, 38.0\% were insulin resistant. Average dairy product intake in the total sample was $2.2 \pm 1.0$ servings per day. Among males, the odds of having insulin resistance were 3.9 times higher $(95 \% \mathrm{Cl} 1.4-11.0$; $p=0.009$ ) for those having a risky waist circumference compared to those having a healthy waist circumference. Among females, being married (OR: $0.2,95 \% \mathrm{Cl} 0.1-0.5 ; p=0.002)$, PBF (OR: 1.2, 95\%Cl 1.0-1.3; $p=0.008)$ and hypertriglyceridemia (OR: 8.7, 95\%Cl: 2.1-35.9; $p=0.003$ ) were associated with HOMA-IR, after controlling for confounders. Dairy intake was not associated with HOMA-IR neither among males $(p=0.777)$, nor among females $(p=0.968)$, after controlling for confounders.

* Correspondence: jelhayek@ndu.edu.lb

'Department of Nursing and Health Sciences, Notre Dame University Louaize (NDU), Zouk Mosbeh, Lebanon

Full list of author information is available at the end of the article

C C The Author(s). 2020 Open Access This article is licensed under a Creative Commons Attribution 4.0 International License, which permits use, sharing, adaptation, distribution and reproduction in any medium or format, as long as you give appropriate credit to the original author(s) and the source, provide a link to the Creative Commons licence, and indicate if changes were made. The images or other third party material in this article are included in the article's Creative Commons licence, unless indicated otherwise in a credit line to the material. If material is not included in the article's Creative Commons licence and your intended use is not permitted by statutory regulation or exceeds the permitted use, you will need to obtain permission directly from the copyright holder. To view a copy of this licence, visit http://creativecommons.org/licenses/by/4.0/. The Creative Commons Public Domain Dedication waiver (http://creativecommons.org/publicdomain/zero/1.0/) applies to the data made available in this article, unless otherwise stated in a credit line to the data. 
(Continued from previous page)

Conclusion: Dairy consumption was not associated with increased insulin resistance. More research focusing on the relationship between dairy intake and insulin resistance is needed, especially in the Arab and Middle-Eastern region. Future studies should examine the effect of different types of dairy products and the effect of different nutrients in dairy products on insulin resistance.

Keywords: Dairy products, Insulin resistance, Homeostatic model assessment for insulin resistance, Cross-sectional, Human subjects

\section{Background}

According to the International Diabetes Federation, the number of people with diabetes in the world is expected to increase from 463 million in 2019 to 700 million in 2045 , with $83.9 \%$ of cases occurring in low and middle income countries [1]. In Lebanon, World Health Organization (WHO) statistics showed that diabetes is standing as the fourth leading cause of death (4\%) with a prevalence rate of $12.6 \%$ [2], higher than that reported by the United States (US) (9.4\%) [3].

Type 2 diabetes, the most common form of diabetes, is mainly caused by insulin resistance [4]. Insulin resistance can be defined as a condition in which the pancreas is required to secrete more insulin than normal in order to achieve normal blood glucose levels due to reduced sensitivity or responsiveness of tissues to insulin biologic activity [5]. The prevalence of insulin resistance varies across countries. Studies showed that it is estimated to be the lowest among European adults with a prevalence of $15.5 \%$ [6], while higher prevalence rates were reported in other countries reaching 23.3, 39.1 and $46.5 \%$ in Thailand, Texas-US and Venezuela [7-9], respectively. Lebanon reported one of the highest prevalence rates compared to other countries reaching $44.6 \%$ among a national sample of 308 adults with an average age of $41.0 \pm 15.5$ years [10].

Several factors could increase the likelihood to develop insulin resistance. For instance, age increases the risk of having insulin resistance due to the high proportion of visceral fat, oxidative stress and mitochondrial dysfunction [11, 12]. Abdominal adiposity and increased body fat are other risk factors for insulin resistance $[13,14]$ and this is due to the high amount of free fatty acids and pro-inflammatory cytokines released from visceral fat tissue into the portal vein of obese subjects, causing the development of hepatic insulin resistance and type 2 diabetes [15]. Other risk factors include gender [16-18], physical inactivity $[19,20]$.

Further, diet has been shown to be effective in improving insulin resistance [21] and reducing the incidence of type 2 diabetes [22]. A study conducted among subjects with impaired glucose tolerance showed that diet was able to reduce the incidence of type 2 diabetes by $33 \%$ $(p<0.003)$, after a follow up period of 6 years [22]. In a study conducted among overweight and obese middle aged women, diet alone was able to reduce insulin resistance by $24 \%$ [21]. Accordingly, more research is focusing on the role of specific food groups in improving insulin resistance.

The consumption of dairy products makes an important contribution to the human diet. They were found to provide more calcium, protein, magnesium, potassium, zinc, and phosphorus per calorie than any other type of food [23]. In Lebanon, as well as in other countries in the Levant region such as Jordan and Syria, dairy products constitute an important part of the traditional food heritage, typically in the fermented form like yogurt, labneh and white cheese [24]. However, not only in Lebanon, but among all Middle Eastern countries, globalization has led to a decreased consumption of dairy products over time $[25,26]$ and a shift to a Western diet including high consumption of fast foods as a result of rapid economic and social changes. These dietary changes were found to be major contributors to central obesity and insulin resistance resulting in an increase in the epidemic of type 2 diabetes [27].

Typical predictors of insulin resistance such as age, gender, physical inactivity, abdominal adiposity and body fat mass have been studied thoroughly in the literature, yet other factors such as dairy products were understudied. To date, few epidemiological studies examined the association between dairy product consumption and insulin resistance; and results are still controversial and require further assessment [28-34]. To our knowledge, no study has examined this association in the Arab and Middle Eastern Region, particularly in Lebanon. It is likely that this association could be different by ethnicity due to different genetic predisposition for diabetes [35]. All of these factors highlight the importance of examining this association in Lebanon. Accordingly, the aim of the study was to investigate the prevalence of insulin resistance among a sample of Lebanese adults, to identify its risk factors depending on gender, and to evaluate the association between insulin resistance and dairy products consumption.

\section{Methods}

\section{Study design and recruitment methods}

A cross-sectional study was carried out on Notre Dame University (NDU) employees in the Zouk Mosbeh, 
North, and Shouf campus between October 2016 and March 2017. The detailed study methodology was previously published [36]. Taking into account the prevalence of insulin resistance of $44.6 \%$ in the Lebanese population [10], a confidence interval of $95 \%$ and a margin of error (d) of 5.5\%, the calculated sample size was estimated to be 316 participants.

Among 600 contacted employees in the three NDU campuses, 360 accepted to participate. Exclusion criteria included pregnancy and lactation, cardiovascular disease (stroke, heart failure and heart attack), diabetes, failure to complete the questionnaires, and presence of a pacemaker or metal pieces in the participant's body. Those who were found to be eligible $(n=286)$ were contacted by the study investigators to arrange for a 30 -min faceto-face interview. All subjects signed informed consent before participating in the study. The study protocol was approved by the Institutional Review Board of Notre Dame University and performed in accordance with the Helsinki Declaration.

\section{Data collection procedures Questionnaires}

During the 30-min face-to-face interview, trained nutritionists filled out four questionnaires: background questionnaire, International Physical Activity Questionnaire, food frequency questionnaire, and a 24-h Multiple-Pass Method recall. All questionnaires were pre-tested using a sample of thirty NDU employees in the three campuses. Revisions and corrections were performed before initializing the study. Details about the background questionnaire (available as a supplementary file [Additional file 1]) and International Physical Activity Questionnaire [37] were previously described [36].

\section{Food frequency questionnaire (FFQ)}

Dairy products intake was assessed using a FFQ that was previously developed by study investigators [38] and adapted to the Lebanese population, including different types of dairy products (milk, yogurt, cheese, ice cream, and labneh). For each food item, participants were asked to mark their frequency of intake of a designated serving/portion size per day/week/months or rarely/never during the past year.

\section{4- $h$ multiple-pass method recall}

A 24-h Multiple-Pass Method recall was used to estimate energy and nutrient intake. It consisted of a fivestep approach conducted in person by a trained nutritionist. Step 1 started with a Quick List where respondents list all foods consumed in a 24-h period. Step 2 included a series of questions that probe for foods that were commonly forgotten during Step 1 including beverages, alcoholic beverages, sweets, savory snacks, fruits, vegetables or cheese, breads or rolls, and one for any additional food not previously mentioned. Step 3 collected the time each food was eaten and the eating occasion. Step 4 was the Detail Cycle where descriptions were obtained for each food reported, along with quantities consumed and where the food was consumed. Step 5 was a final review question, the Final Probe, which provided the respondent a last opportunity to recall any foods that had not been previously reported in the interview [39].

\section{Energy and nutrient intake}

The Nutritionist Pro diet analysis software, version 31.0 (Axxya Systems, Woodinville, WA, US), was used to create estimates of energy and different nutrients' intake.

\section{Anthropometric measurements}

Anthropometric measurements including height, waist circumference, body weight were taken and body composition was assessed using BIA machine InBody 720 (Biospace, Seoul, Korea). Details about anthropometric measurements was previously published [36].

\section{Biochemical measurements}

Upon the visit to the nutrition laboratory, a nurse collected a fasting sample of blood. Samples collected at the Shouf and North campuses were transported to the Zouk Mosbeh campus on ice. Samples were stored at $-20^{\circ} \mathrm{C}$ in the freezer for a maximum of 6 weeks before analysis. Biochemical assessment included serum triglycerides (TG), total cholesterol, high density lipoproteins (HDL) cholesterol, low density lipoproteins (LDL) cholesterol, creactive protein (CRP), and fasting blood glucose which were measured using a dry chemistry analyzer Vitros 250 (Ortho Clinical Diagnostic, Raritan, New Jersey, US) available at the Biology laboratory at the Zouk Mosbeh campus. Insulin levels were measured by enzyme linked immunosorbent assay (ELISA) technique using insulin specific kits (Labor Diagnostika Nord, Nordhorn, Germany) with sensitivity of $1.76 \mu \mathrm{IU} / \mathrm{ml}$.

\section{Homoeostatic model assessment - insulin resistance (HOMA-IR)}

Insulin resistance was assessed using HOMA-IR, one of the most widely used indices based on fasting parameters [40]. It is considered to be a reliable surrogate measure of in vivo insulin sensitivity in humans [41] as well as a practical and convenient tool [42]. The HOMA-IR formula used was the following:

HOMA-IR $=\{$ [fasting insulin $(\mu \mathrm{U} / \mathrm{mL})] \times[$ fasting glucose $(\mathrm{mmol} / \mathrm{L})]\} / 22.5$ [40]. A person with HOMA-IR levels $\geq 2.5$ was considered to be insulin resistant [43]. 


\section{Statistical analyses}

Quantitative and qualitative measurements were summarized as mean \pm standard deviation and $\mathrm{n}(\%)$, respectively. Comparisons of continuous and categorical variables were performed using independent sample $\mathrm{T}$ Test (across 2 groups) - One Way Analysis of Variance (ANOVA)/ Kruskal Wallis test (across $>2$ groups) test and the chi square test, respectively. Two logistic regression models, stratified by gender (because of interaction between gender and body mass index (BMI), gender and TG), were performed, where HOMA-IR categories (categorical) were used as the dependent variable and dairy intake (continuous) was used as the main independent variable, controlling for important confounders having a $p$-value $<0.20$ among males and a $p$-value $<0.05$ among females in the bivariate analysis, in addition to age. Among males, these confounders included education, income, BMI, PBF, risky waist circumference, physical activity (PA) and TG levels. However, education and income were removed from the model because of high correlation $(\rho>0.80)$. Among females, confounders that were controlled for were marital status, BMI, PBF, risky waist circumference, TG, HDL and CRP levels. Statistical analyses were performed using the Statistical Package for Social Sciences (SPSS) version 23 for Windows. A $p$ value of less than 0.05 was considered statistically significant.

\section{Results}

A total of 286 subjects ( $46.9 \%$ men and $53.1 \%$ women) with a mean age of $41.2 \pm 11.0$ years participated in the study. Characteristics of study population stratified by gender were summarized in Table 1 . The majority of participants were healthy, not suffering from medical morbidity (65.4\%), non-smokers (63.6\%), and did not drink alcohol (75.2\%). More than half of the participants (64.0\%) had a low level of PA. Subjects were primarily married (63.3\%), holding a university degree (80.1\%), with an income of less than $\$ 4000$ per month (58.0\%). More than one third of the participants were of normal weight (39.2\%), while the majority was overweight and obese (60.1\%) (Table 1). Mean glucose and insulin concentrations were $5.0 \pm 0.9 \mathrm{mmol} / \mathrm{L}$ and $12.1 \pm 8.0 \mathrm{uIU} /$ $\mathrm{mL}$, respectively. In the total sample, more than one third of participants (38.0\%) were insulin resistant (Fig. 1).

The average dairy product intake in the total sample was $2.2 \pm 1.0$ servings per day. More than $80 \%$ of participants were not meeting the recommendation of $\geq 3$ servings of dairy/ day with no statistically significant difference across genders ( $p=0.557$ ) (Fig. 2).

Across genders, men were significantly older $(43.7 \pm$ 11.8 years) than women (age: $39.0 \pm 9.7$ years) $(p<0.001)$. More men $(45.5 \%)$ were smokers $(p=0.003)$ and alcohol drinkers (33.6\%) compared to women $(28.3 \%$ were smokers and $17.1 \%$ were alcohol drinkers, $p<0.001)$. More than half of women were of a healthy weight (57.2\%) whereas more than half of men were overweight $(51.5 \%)$ and obese $(29.9 \%)(p<0.001)$. Men had significantly lower PBF $(27.1 \pm 6.7 \%)$ than women (PBF: $33.7 \pm$ $7.7 \%, p<0.001)$. Men had higher glucose levels $(5.3 \pm$ $1.2 \mathrm{mmol} / \mathrm{L})$ than women $4.8 \pm 0.5 \mathrm{mmol} / \mathrm{L}, p<0.001)$ (Table 1). A higher proportion of men $(47.0 \%)$ were insulin resistant compared to women $(31.6 \%, p<0.01)$ (Fig. 1).

Table 2 summarizes the socio-demographic, dietary, lifestyle, anthropometric, and biochemical factors associated with dairy consumption. A higher proportion of married participants $(75.3 \%)$ were in quartile 3 of dairy intake compared to quartile $4(50.7 \%, p=0.024)$ while a higher proportion of single participants were in quartile 4 (49.3\%) of dairy intake. Individuals in quartile 3 of dairy product intake had lower BMI $\left(25.6 \pm 4.3 \mathrm{~kg} / \mathrm{m}^{2}\right)$, PBF $(28.4 \pm 7.1 \%)$ and waist circumference $(92.8 \pm 11.6$ $\mathrm{cm}$ ) than individuals in quartile 2 (BMI: $28.0 \pm 5.2 \mathrm{~kg} /$ $\mathrm{m}^{2}, p=0.021$; PBF: $31.8 \pm 8.1 \%, p=0.039$; waist circumference: $98.8 \pm 12.0 \mathrm{~cm}, p=0.019)$. Subjects in the lowest quartile of dairy intake had lower intake of calcium $(619.5 \pm 435.2 \mathrm{mg})$, magnesium $(248.5 \pm 142.0 \mathrm{mg})$ and potassium (2294.5 $\pm 1049.1 \mathrm{mg})$ compared to individuals with the highest intake of dairy (calcium: $887.4 \pm 525.3$ $\mathrm{mg}, p=0.011$; magnesium: $319.7 \pm 154.4 \mathrm{mg}, p=0.025$; potassium: $2890.8 \pm 1288.1 \mathrm{mg}, p=0.026$ ). Normal TG levels were more common among individuals in quartile 3 of dairy intake $(78.1 \%)$ compared to individuals in quartile $2(57.1 \%)(\mathrm{p}=0.025)$ (Table 2$)$.

Table 3 summarizes the socio-demographic, dietary, lifestyle, anthropometric, and biochemical factors associated with HOMA-IR across genders. A higher proportion of obese males (42.9\%) were insulin resistant compared to those who weren't $(18.3 \%)(p<0.001)$. In addition, males with HOMA-IR $\geq 2.5$ had significantly higher PBF $(29.4 \pm 6.2 \%)$ compared to those with HOMA-IR $<2.5(25.0 \pm 6.5 \%, p<0.001)$. A higher proportion of males having risky waist circumference were insulin resistant (66.7\%), compared to those having a healthy waist circumference $(25.4 \%)(p<0.001)$. Furthermore, males with HOMA-IR $\geq 2.5$ had higher glucose $(5.6 \pm 1.6)$ and insulin levels $(16.3 \pm 8.2)$ compared to those with HOMA-IR $<2.5$ (glucose: $5.0 \pm 0.5, p=0.003$, insulin: $8.8 \pm 1.3, p<0.001$ ).

Among females, a higher proportion of married females $(69.2 \%)$ were insulin resistant compared to those who weren't $(41.7 \%, p<0.001)$. Moreover, a higher proportion of obese females were insulin resistant (35.4\%) than non-insulin resistant $(7.7 \%, \mathrm{p}<0.001)$. In addition, females with HOMA-IR $\geq 2.5$ had significantly higher PBF $(38.7 \pm 7.5 \%)$ compared to those with HOMA-IR < 
Table 1 Sample characteristics (socio-demographic, dietary, lifestyle, anthropometric, and biochemical factors)

\begin{tabular}{|c|c|c|c|c|c|c|c|}
\hline \multirow[b]{2}{*}{ Characteristics } & \multicolumn{2}{|c|}{ Total $(n=286)$} & \multicolumn{2}{|c|}{ Men $(n=134)$} & \multicolumn{2}{|c|}{ Women $(n=152)$} & \multirow[b]{2}{*}{$P$-value } \\
\hline & $\mathrm{n}$ or mean & $\%$ or SD & $\mathrm{n}$ or mean & $\%$ or SD & $\mathrm{n}$ or mean & $\%$ or SD & \\
\hline Age (years) & 41.2 & 11.0 & 43.7 & 11.8 & 39.0 & 9.7 & $<0.001$ \\
\hline \multicolumn{8}{|l|}{ Medical morbidity } \\
\hline - No & 187 & 65.4 & 83 & 61.9 & 104 & 68.4 & \multirow[t]{2}{*}{0.250} \\
\hline - Yes & 99 & 34.6 & 51 & 38.1 & 48 & 31.6 & \\
\hline \multicolumn{8}{|l|}{ Smoking } \\
\hline - No & 182 & 63.6 & 73 & 54.5 & 109 & 71.7 & \multirow[t]{2}{*}{0.003} \\
\hline - Yes & 104 & 36.4 & 61 & 45.5 & 43 & 28.3 & \\
\hline \multicolumn{8}{|l|}{ Alcohol drinking } \\
\hline$-\mathrm{No}$ & 215 & 75.2 & 89 & 66.4 & 126 & 82.9 & \multirow[t]{2}{*}{$<0.001$} \\
\hline - Yes & 71 & 24.8 & 45 & 33.6 & 26 & 17.1 & \\
\hline \multicolumn{8}{|l|}{ PA ${ }^{1}$ level } \\
\hline - Low & 183 & 64.0 & 82 & 61.2 & 101 & 66.4 & \multirow[t]{2}{*}{0.356} \\
\hline - Moderate/High & 103 & 36.0 & 52 & 38.8 & 51 & 33.6 & \\
\hline \multicolumn{8}{|l|}{ Marital status } \\
\hline - Single/ Separated/ Divorced & 105 & 36.7 & 45 & 33.6 & 60 & 39.5 & \multirow[t]{2}{*}{0.302} \\
\hline - Married & 181 & 63.3 & 89 & 66.4 & 92 & 60.5 & \\
\hline \multicolumn{8}{|l|}{ Education level } \\
\hline - High school & 57 & 19.9 & 37 & $27.6^{\mathrm{a}}$ & 20 & $13.2^{\mathrm{a}}$ & \multirow[t]{3}{*}{0.003} \\
\hline - Bachelor degree & 76 & 26.6 & 27 & $20.1^{\mathrm{a}}$ & 49 & $32.2^{\mathrm{a}}$ & \\
\hline - Graduate & 153 & 53.5 & 70 & 52.2 & 83 & 54.6 & \\
\hline \multicolumn{8}{|l|}{ Income (\$) } \\
\hline$-<2250$ & 85 & 29.7 & 44 & 32.8 & 41 & 27.0 & \multirow[t]{3}{*}{0.109} \\
\hline$-2250-4000$ & 81 & 28.3 & 30 & 22.4 & 51 & 33.6 & \\
\hline$->4000$ & 120 & 42.0 & 60 & 44.8 & 60 & 39.5 & \\
\hline \multicolumn{8}{|l|}{$B M I^{2}$} \\
\hline - Underweight & 2 & 0.7 & 0 & 0.0 & 2 & 1.3 & \multirow[t]{4}{*}{$<0.001$} \\
\hline - Normal & 112 & 39.2 & 25 & $18.7^{\mathrm{a}}$ & 87 & $57.2^{\mathrm{a}}$ & \\
\hline - Overweight & 107 & 37.4 & 69 & $51.5^{\mathrm{a}}$ & 38 & $25.0^{\mathrm{a}}$ & \\
\hline - Obese & 65 & 22.7 & 40 & $29.9^{\mathrm{a}}$ & 25 & $16.4^{\mathrm{a}}$ & \\
\hline $\mathrm{PBF}^{3}$ & 30.6 & 8.0 & 27.1 & 6.7 & 33.7 & 7.7 & $<0.001$ \\
\hline \multicolumn{8}{|l|}{ Waist circumference risky ${ }^{4}$} \\
\hline$-\mathrm{No}$ & 152 & 53.1 & 74 & 55.2 & 78 & 51.3 & \multirow[t]{2}{*}{0.509} \\
\hline - Yes & 134 & 46.9 & 60 & 44.8 & 74 & 48.7 & \\
\hline Calories (Cal) & 1942.2 & 811.5 & 2165.9 & 944.3 & 1744.9 & 611.6 & $<0.001$ \\
\hline Dairy product intake (serving/ day) & 2.2 & 1.0 & 2.2 & 1.0 & 2.2 & 1.0 & 0.999 \\
\hline Glucose (mmol/L) & 5.0 & 0.9 & 5.3 & 1.2 & 4.8 & 0.5 & $<0.001$ \\
\hline Insulin (ulU/ ml) & 12.1 & 8.0 & 12.3 & 6.8 & 11.8 & 8.9 & 0.604 \\
\hline Hypertriglyceridemia & & & & & & & \\
\hline - Normal TG levels & 204 & 71.3 & 75 & 56.0 & 129 & 84.9 & $<0.001$ \\
\hline - Hypertriglyceridemia & 82 & 28.7 & 59 & 44.0 & 23 & 15.1 & \\
\hline Cholesterol $^{6}$ & & & & & & & \\
\hline - Desirable & 185 & 64.7 & 90 & 67.2 & 95 & 62.5 & 0.410 \\
\hline - High & 101 & 35.3 & 44 & 32.8 & 57 & 37.5 & \\
\hline
\end{tabular}


Table 1 Sample characteristics (socio-demographic, dietary, lifestyle, anthropometric, and biochemical factors) (Continued)

\begin{tabular}{|c|c|c|c|c|c|c|c|}
\hline \multirow[b]{2}{*}{ Characteristics } & \multicolumn{2}{|c|}{ Total $(n=286)$} & \multicolumn{2}{|c|}{ Men $(n=134)$} & \multicolumn{2}{|c|}{ Women $(n=152)$} & \multirow[b]{2}{*}{$P$-value } \\
\hline & n or mean & $\%$ or SD & $\mathrm{n}$ or mean & $\%$ or SD & $\mathrm{n}$ or mean & $\%$ or SD & \\
\hline \multicolumn{8}{|l|}{$\mathrm{HDL}^{7}$} \\
\hline - Normal & 219 & 76.6 & 100 & 74.6 & 119 & 78.3 & 0.466 \\
\hline - Low & 67 & 23.4 & 34 & 25.4 & 33 & 21.7 & \\
\hline \multicolumn{8}{|l|}{$\mathrm{LDL}^{8}$} \\
\hline - Optimal & 98 & 34.5 & 47 & 35.6 & 51 & 33.6 & 0.717 \\
\hline - Above optimal & 186 & 65.5 & 85 & 64.4 & 101 & 66.4 & \\
\hline \multicolumn{8}{|l|}{ CRP $^{9}$} \\
\hline - Low/ moderate & 124 & 43.4 & 46 & 34.3 & 78 & 51.3 & 0.004 \\
\hline - High & 162 & 56.6 & 88 & 65.7 & 74 & 48.7 & \\
\hline
\end{tabular}

${ }^{1}$ Physical activity

${ }^{2}$ Body Mass Index - BMI $<18.5 \mathrm{~kg} / \mathrm{m}^{2}$, normal weight $18.5-24.9 \mathrm{~kg} / \mathrm{m}^{2}$, overweight $25-29.9 \mathrm{~kg} / \mathrm{m}^{2}$, and obese $\geq 30 \mathrm{~kg} / \mathrm{m}^{2}$ [44]

${ }^{3}$ Percent body fat

${ }^{4}$ Risky waist circumference: $>102 \mathrm{~cm}$ in men, $>88 \mathrm{~cm}$ in women [44]

${ }^{5}$ Triglycerides - Normal levels: $<150 \mathrm{mg} / \mathrm{dL}$ [45]

${ }^{6}$ Desirable levels: $<200 \mathrm{mg} / \mathrm{dL}$ [45]

${ }^{7}$ High Density Lipoprotein - Normal levels: $\geq 40 \mathrm{mg} / \mathrm{dL}, \geq 50 \mathrm{mg} / \mathrm{dL}$ [45]

${ }^{8}$ Low Density Lipoprotein - Optimal levels: $<100 \mathrm{mg} / \mathrm{dL}$ [45]

${ }^{9}$ C-Reactive Protein - Low/ moderate levels: $<3 \mathrm{mg} / \mathrm{L}$ [46]

Columns with superscripts without a common symbol differ, $P<0.05$

$2.5(31.4 \pm 6.6 \%, p<0.001)$. A higher proportion of females having risky waist circumference were insulin resistant (77.1\%), compared to those having a healthy waist circumference $(35.6 \%, p<0.001)$. Furthermore, females with HOMA-IR $\geq 2.5$ had higher glucose (5.1 \pm $0.6)$ and insulin levels $(19.0 \pm 13.1)$ compared to those with HOMA-IR $<2.5$ (glucose: $4.7 \pm 0.4, p<0.001$, insulin: $8.5 \pm 1.6, \mathrm{p}<0.001)$. Normal TG $(95.2 \%)$ and HDL levels (83.7\%) and low/moderate CRP levels (58.7\%) were more common among individuals who weren't insulin resistant compared to those who were (normal TG: $62.5 \%, p<0.001$, normal HDL: $66.7 \%, p=0.018$ and low/ moderate CRP: $35.4 \%, p=0.008$ ) (Table 3).

Tables 4 and 5 examined the association between dairy intake and HOMA-IR, after controlling for confounding variables among males and females, respectively. Among males, the odds of having insulin resistance were 3.9 times higher $(95 \%$ CI $1.4-11.0 ; p=0.009)$ for those having a risky waist circumference compared to those having a healthy waist circumference. Among females, being married had lower odds of having insulin resistance than single females (OR: 0.2, 95\% CI 0.1-0.5, $p=0.002$ ). As PBF increased by 1 unit, the odds of having insulin resistance increased by 1.2 times $(95 \%$ CI $1.0-1.3, p=$ 0.008). Finally, the likelihood of having insulin resistance were 8.7 times higher among those suffering from hypertriglyceridemia compared to those with normal TG levels $(95 \%$ CI: $2.1-35.9, p=0.003)$. Dairy product intake was not associated with HOMA-IR neither among males (95\%CI $0.6-1.4, p=0.777)$ nor among females $(95 \% \mathrm{CI}$

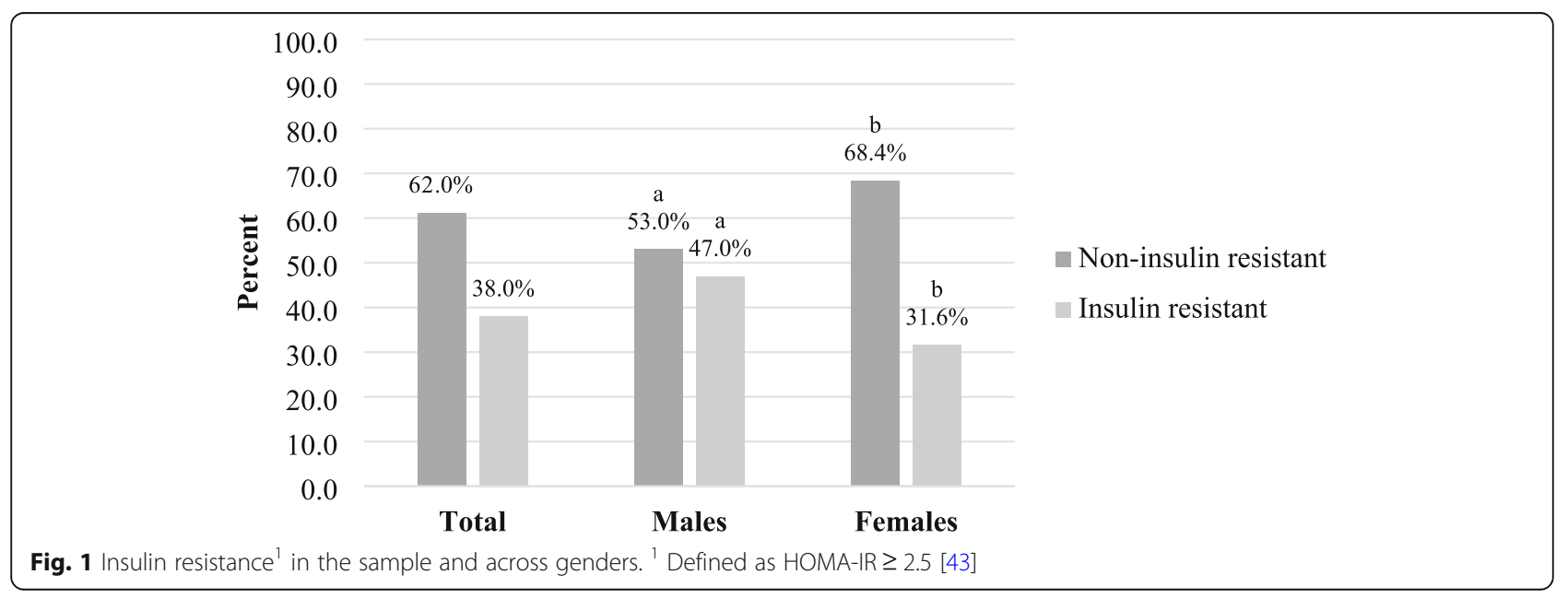




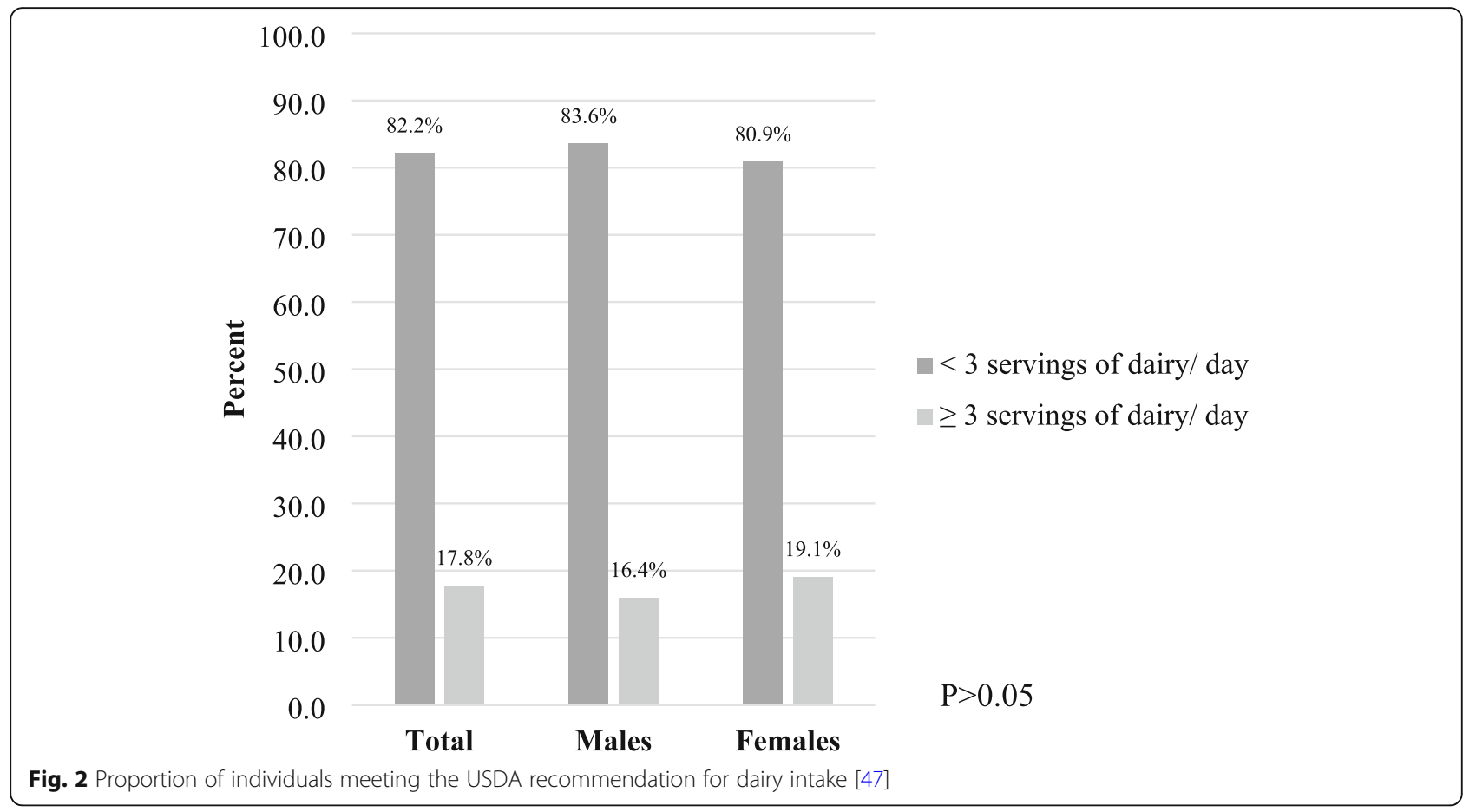

0.6-1.6, $p=0.968$ ), after controlling for important confounding variables (Tables 4 and 5).

\section{Discussion}

The present study showed that the prevalence of insulin resistance is high in a sample of Lebanese adults, with a higher prevalence among men as compared to women. Dairy product intake was not significantly associated with insulin resistance.

In our study, the prevalence of insulin resistance was $38.0 \%$. Our findings are in line with another local study by Naja et al., 2012 (44.6\%), among a national random sample of 308 Lebanese adults [10] with similar characteristics of the study populations including mean age, gender distribution, average BMI, and smoking status. Internationally, the prevalence of insulin resistance among adults varies across different countries, ranging from $15.5 \%$ in Denmark [6] to $46.5 \%$ in Venezuela [9]. Higher prevalence rate of insulin resistance is reported in Lebanon since the Arab ethnicity is associated with higher risk of developing type 2 diabetes mellitus when compared with other ethnicities [48].

In the current study, the prevalence of insulin resistance was higher among men compared to women, while Naja et al., 2012 did not report on gender differences, other studies did report similar trends [6, 49]. The findings of our study are concordant with those of Friedrich et al., 2012, since in both studies, male participants had significantly higher age and BMI than women, which could explain the difference in prevalence rates of insulin resistance.

The average dairy product intake in this study was $2.2 \pm 1.0$ servings/day which was in line with another study conducted in Lebanon by Nasreddine et al., 2006 that reported a consumption of 2.06 servings per day among a random sample of 444 Lebanese adults aged between 25 and 54 years, recruited in Beirut and its suburbs [25]. In contrast, Farhat et al. 2016 reported a lower consumption of dairy products of 1.4 servings/day among a convenient sample of 615 adults aged between 19 to 70 years recruited from different regions across Lebanon [26]. The average consumption of dairy products differed between studies due to difference in the study populations including different age ranges and areas from which the samples were selected and different dietary assessment tools.

The consumption of dairy products was below the recommendation of 3 cups per day set by the United States Department of Agriculture (USDA) myplate [47] and the Lebanese Food-Based Dietary Guidelines [50]. Several countries in the Middle East reported dairy product consumption below the USDA myplate recommendation $[51,52]$. For instance, the average intake of dairy products was $2.2 \pm 1.1$ servings/ day among a sample of 126 Emirati adults with an average age of $37.0 \pm 11$ years [51]. Further, lower intakes of dairy products $(0.7-0.85$ servings/day) have been reported among a sample of 486 Iranian women aged between 40 and 60 years [52]. 
Table 2 Socio-demographic, dietary, lifestyle, anthropometric, and biochemical factors associated with dairy intake

\begin{tabular}{|c|c|c|c|c|c|c|c|c|c|}
\hline \multirow{3}{*}{ Characteristic } & \multicolumn{8}{|c|}{ Dairy intake quartiles } & \multirow[b]{3}{*}{$P$-value } \\
\hline & \multicolumn{2}{|c|}{$\begin{array}{l}\text { Quartile } 1 \text { ( } \leq 1.55 \text { serv/d) } \\
n=72\end{array}$} & \multicolumn{2}{|c|}{$\begin{array}{l}\text { Quartile } 2(1.56-2.15 \text { serv/d) } \\
n=70\end{array}$} & \multicolumn{2}{|c|}{$\begin{array}{l}\text { Quartile } 3 \text { ( } 2.16-2.73 \text { serv/d) } \\
n=73\end{array}$} & \multicolumn{2}{|c|}{$\begin{array}{l}\text { Quartile } 4 \text { ( } \geq 2.74 \text { serv/ d) } \\
n=71\end{array}$} & \\
\hline & $\mathrm{n}$ or mean & $\%$ or SD & $\mathrm{n}$ or mean & $\%$ or SD & $\mathrm{n}$ or mean & $\%$ or SD & $\mathrm{n}$ or mean & $\%$ or SD & \\
\hline Age (years) & 42.3 & 12.3 & 42.6 & 12.1 & 39.9 & 9.6 & 40.1 & 9.5 & 0.443 \\
\hline \multicolumn{10}{|l|}{ Gender } \\
\hline - Male & 32 & 44.4 & 36 & 51.4 & 36 & 49.3 & 30 & 42.3 & \multirow[t]{2}{*}{0.674} \\
\hline - Female & 40 & 55.6 & 34 & 48.6 & 37 & 50.7 & 41 & 57.7 & \\
\hline \multicolumn{10}{|l|}{ Marital status } \\
\hline $\begin{array}{l}\text { - Single/ Separated/ } \\
\text { Divorced }\end{array}$ & 26 & 36.1 & 26 & 37.1 & 18 & $24.7^{\mathrm{a}}$ & 35 & $49.3^{\mathrm{a}}$ & \multirow[t]{2}{*}{0.024} \\
\hline - Married & 46 & 63.9 & 44 & 62.9 & 55 & $75.3^{\mathrm{a}}$ & 36 & $50.7^{\mathrm{a}}$ & \\
\hline \multicolumn{10}{|l|}{ Education level } \\
\hline - High school & 17 & 23.6 & 14 & 20.0 & 12 & 16.4 & 14 & 19.7 & \multirow[t]{3}{*}{0.446} \\
\hline - Bachelor degree & 13 & 18.1 & 24 & 34.3 & 21 & 28.8 & 18 & 25.4 & \\
\hline - Graduate & 42 & 58.3 & 32 & 45.7 & 40 & 54.8 & 39 & 54.9 & \\
\hline \multicolumn{10}{|l|}{ Income (\$) } \\
\hline$-<2250$ & 17 & 23.6 & 23 & 32.9 & 18 & 24.7 & 27 & 38.0 & \multirow[t]{3}{*}{0.293} \\
\hline$-2250-4000$ & 19 & 26.4 & 19 & 27.1 & 21 & 28.8 & 22 & 31.0 & \\
\hline$->4000$ & 36 & 50.0 & 28 & 40.0 & 34 & 46.6 & 22 & 31.0 & \\
\hline \multicolumn{10}{|l|}{ Medical morbidity } \\
\hline - No & 42 & 58.3 & 41 & 58.6 & 49 & 67.1 & 55 & 77.5 & \multirow[t]{2}{*}{0.053} \\
\hline - Yes & 30 & 41.7 & 29 & 41.4 & 24 & 32.9 & 16 & 22.5 & \\
\hline \multicolumn{10}{|l|}{$\mathrm{PA}^{1}$ level } \\
\hline - Low & 46 & 63.9 & 47 & 67.1 & 47 & 64.4 & 43 & 60.6 & \multirow[t]{2}{*}{0.880} \\
\hline - Moderate/ High & 26 & 36.1 & 23 & 32.9 & 26 & 35.6 & 28 & 39.4 & \\
\hline $\mathrm{BMI}^{2}\left(\mathrm{Kg} / \mathrm{m}^{2}\right)$ & 26.1 & 4.3 & $28.0^{\mathrm{a}}$ & 5.2 & $25.6^{\mathrm{a}}$ & 4.3 & 27.0 & 5.2 & 0.021 \\
\hline $\mathrm{PBF}^{3}$ & 31.5 & 8.2 & $31.8^{\mathrm{a}}$ & 8.1 & $28.4^{\mathrm{a}}$ & 7.1 & 30.8 & 8.1 & 0.039 \\
\hline Waist circumference $(\mathrm{cm})$ & 94.8 & 10.6 & $98.8^{\mathrm{a}}$ & 12.0 & $92.8^{\mathrm{a}}$ & 11.6 & 94.7 & 12.5 & 0.019 \\
\hline Calcium (mg) & $619.5^{a}$ & 435.2 & 744.0 & 415.7 & 815.4 & 595.7 & $887.4^{a}$ & 525.3 & 0.011 \\
\hline Magnesium (mg) & $248.5^{\mathrm{a}}$ & 142.0 & 264.2 & 140.5 & 285.6 & 148.3 & $319.7^{\mathrm{a}}$ & 154.4 & 0.025 \\
\hline Potassium (mg) & $2294.5^{a}$ & 1049.1 & 2474.7 & 1143.2 & 2617.3 & 1308.5 & $2890.8^{\mathrm{a}}$ & 1288.1 & 0.026 \\
\hline Glucose (mmol/L) & 5.0 & 0.5 & 5.2 & 1.3 & 5.0 & 0.5 & 5.0 & 1.1 & 0.363 \\
\hline Insulin (ulU/ ml) & 12.1 & 6.2 & 12.9 & 10.0 & 11.3 & 7.1 & 12.0 & 8.3 & 0.729 \\
\hline \multicolumn{10}{|l|}{$\mathrm{HOMA}-\mathrm{IR}^{4}$} \\
\hline - Non-insulin resistant & 43 & 59.7 & 41 & 58.6 & 47 & 64.4 & 44 & 62.0 & \multirow[t]{2}{*}{0.897} \\
\hline - Insulin resistant & 29 & 40.3 & 29 & 41.4 & 26 & 35.6 & 27 & 38.0 & \\
\hline \multicolumn{10}{|l|}{ Hypertriglyceridemia } \\
\hline - Normal TG5 levels & 54 & 75.0 & 40 & $57.1^{\mathrm{a}}$ & 57 & $78.1^{\mathrm{a}}$ & 53 & 74.6 & 0.025 \\
\hline - Hypertriglyceridemia & 18 & 25.0 & 30 & $42.9^{a}$ & 16 & $21.9^{\mathrm{a}}$ & 18 & 25.4 & \\
\hline Cholesterol $^{6}$ & & & & & & & & & \\
\hline - Desirable & 44 & 61.1 & 40 & 57.1 & 51 & 69.9 & 50 & 70.4 & 0.259 \\
\hline - High & 28 & 38.9 & 30 & 42.9 & 22 & 30.1 & 21 & 29.6 & \\
\hline $\mathrm{HDL}^{7}$ & & & & & & & & & \\
\hline - Normal & 55 & 76.4 & 49 & 70.0 & 62 & 84.9 & 53 & 74.6 & 0.197 \\
\hline - Low & 17 & 23.6 & 21 & 30.0 & 11 & 15.1 & 18 & 25.4 & \\
\hline
\end{tabular}


Table 2 Socio-demographic, dietary, lifestyle, anthropometric, and biochemical factors associated with dairy intake (Continued)

\begin{tabular}{|c|c|c|c|c|c|c|c|c|c|}
\hline \multirow{3}{*}{ Characteristic } & \multicolumn{8}{|c|}{ Dairy intake quartiles } & \multirow[b]{3}{*}{$P$-value } \\
\hline & \multicolumn{2}{|c|}{$\begin{array}{l}\text { Quartile } 1 \text { ( } \leq 1.55 \text { serv/ d) } \\
n=72\end{array}$} & \multicolumn{2}{|c|}{$\begin{array}{l}\text { Quartile } 2(1.56-2.15 \text { serv/d) } \\
n=70\end{array}$} & \multicolumn{2}{|c|}{$\begin{array}{l}\text { Quartile } 3 \text { (2.16-2.73 serv/d) } \\
n=73\end{array}$} & \multicolumn{2}{|c|}{$\begin{array}{l}\text { Quartile } 4 \text { ( } \geq 2.74 \text { serv/ d) } \\
n=71\end{array}$} & \\
\hline & $\mathrm{n}$ or mean & $\%$ or SD & $\mathrm{n}$ or mean & $\%$ or SD & $\mathrm{n}$ or mean & $\%$ or SD & $\mathrm{n}$ or mean & $\%$ or SD & \\
\hline \multicolumn{10}{|l|}{$\mathrm{LDL}^{8}$} \\
\hline - Optimal & 25 & 34.7 & 19 & 27.5 & 29 & 39.7 & 25 & 35.7 & 0.492 \\
\hline - Above optimal & 47 & 65.3 & 50 & 72.5 & 44 & 60.3 & 45 & 64.3 & \\
\hline \multicolumn{10}{|l|}{ CRP $^{9}$} \\
\hline - Low/ moderate & 29 & 40.3 & 25 & 35.7 & 35 & 47.9 & 35 & 49.3 & 0.309 \\
\hline - High & 43 & 59.7 & 45 & 64.3 & 38 & 52.1 & 36 & 50.7 & \\
\hline
\end{tabular}

${ }^{1}$ Physical activity

${ }^{2}$ Body Mass Index - BMI $<18.5 \mathrm{~kg} / \mathrm{m}^{2}$, normal weight $18.5-24.9 \mathrm{~kg} / \mathrm{m}^{2}$, overweight $25-29.9 \mathrm{~kg} / \mathrm{m}^{2}$, and obese $\geq 30 \mathrm{~kg} / \mathrm{m}^{2}$ [44]

${ }^{3}$ Percent body fat

${ }^{4}$ Homeostatic Model Assessment for Insulin Resistance - HOMA-IR levels $\geq 2.5$ indicates insulin resistance [43]

${ }^{5}$ Triglycerides - Normal levels: $<150 \mathrm{mg} / \mathrm{dL}[45]$

${ }^{6}$ Desirable levels: $<200 \mathrm{mg} / \mathrm{dL}$ [45]

${ }^{7}$ High Density Lipoprotein - Normal levels: $\geq 40 \mathrm{mg} / \mathrm{dL}, \geq 50 \mathrm{mg} / \mathrm{dL}$ [45]

${ }^{8}$ Low Density Lipoprotein - Optimal levels: $<100 \mathrm{mg} / \mathrm{dL}$ [45]

${ }^{9}$ C-Reactive Protein - Low/ moderate levels: $<3 \mathrm{mg} / \mathrm{L}$ [46]

Columns with superscripts without a common symbol differ, $P<0.05$

Several anthropometric measures including BMI, PBF and waist circumference were inversely associated with dairy consumption. Our results were concordant with the literature [53, 54]. For instance, Mimiran et al., 2005 reported a significant inverse correlation between the number of servings of dairy per day and BMI among a sample of 462 healthy Iranians, aged $>16$ years, after controlling for important confounders $(r=-0.38, p<$ 0.05) [53]. Moreover, Shin et al., 2017, reported that those who consumed $\geq 2$ servings of milk per day had lower BMI than those who consumed none or rarely milk $(p<0.0001)$ among 86,738 Korean women aged between 40 and 69 years [54]. Further, Shin et al., 2017, reported that higher milk consumption was associated with reduced odds of hypertriglyceridemia, which was also observed by our study and by others in the literature [54-56]. The mechanism by which dairy consumption affects body weight, fat percentage and triglycerides is not fully elucidated, however few mechanisms were suggested [57, 58]. Dairy products are an important source of calcium and individuals consuming dairy products tend to have higher calcium intakes, as evident by our study and by others [59, 60]. Several studies found an inverse relationship between body weight or body fat and calcium [61-63]. It could be that calcium affects body weight and fat mass through inhibiting fat absorption which has been shown to cause a reduction in TG level [64]. Further, calcium helps in the regulation of adipocyte metabolism, decreasing fatty acid synthesis, increasing lipolysis, and thus depleting TG stores [65].

In our study, a higher proportion of married females were not insulin resistant compared to those who were single. The association between marital status and insulin resistance was not thoroughly investigated in the literature except in one study that showed opposite results [9]. Bermudez et al., 2016 showed that married individuals had higher HOMA-IR values compared to single individuals among 2026 participants with an average age of $39.7 \pm 15.3$ years [9]. In this study, a higher proportion of married participants were males, inactive and had elevated BMI which would explain their results [9]. While in the present study, no statistical difference was observed between single and married females on these same parameters, however married females were more likely to have normal HDL levels $(p=0.043)$, which could explain the association observed in our study.

In our study, participants with higher adiposity were more likely to be insulin resistant. Similar results were observed previously in the literature $[13,14,66]$. In case of high PBF, the liver is directly exposed to free fatty acids and pro-inflammatory cytokines released from visceral fat tissue into the portal vein of obese subjects which will lead to the development of hepatic insulin resistance and type 2 diabetes [15].

Our study found that females with normal TG levels were less likely to be insulin resistant. Likewise, higher risk of hypertriglyceridemia were observed among 990 Thai women aged $\geq 35$ years in the highest quartile of HOMA-IR values compared to those in the lowest quartile [7]. Further, Keska et al., 2013 reported that individuals with higher HOMA-IR had higher TG levels compared to their counterparts with lower HOMA-IR $(p<0.01)$ among 87 young men with an average age of $19.8 \pm 0.8$ years [67].

Our study did not find an association between dairy product intake and insulin resistance, before and after controlling for confounding variables. The association between dairy product intake and insulin resistance is 
Table 3 Socio-demographic, lifestyle, anthropometric, and biochemical factors associated with HOMA-IR

\begin{tabular}{|c|c|c|c|c|c|c|c|c|c|c|}
\hline \multirow{4}{*}{ Characteristic } & \multicolumn{10}{|c|}{ HOMA-IR categories } \\
\hline & \multicolumn{5}{|c|}{ Men $(n=134)$} & \multicolumn{5}{|c|}{ Women $(n=152)$} \\
\hline & \multicolumn{2}{|c|}{$\begin{array}{l}\text { HOMA-IR }<2.5 \\
N=71\end{array}$} & \multicolumn{2}{|c|}{$\begin{array}{l}\text { HOMA-IR } \geq 2.5) \\
N=63\end{array}$} & \multirow[b]{2}{*}{$P$-value } & \multicolumn{2}{|c|}{$\begin{array}{l}\mathrm{HOMA}-\mathrm{IR}<2.5 \\
N=104\end{array}$} & \multicolumn{2}{|c|}{$\begin{array}{l}\text { HOMA-IR } \geq 2.5) \\
N=48\end{array}$} & \multirow[b]{2}{*}{$P$-value } \\
\hline & $\mathrm{n}$ or mean & $\%$ or SD & $\mathrm{n}$ or mean & $\%$ or SD & & $\mathrm{n}$ or mean & $\%$ or SD & $\mathrm{n}$ or mean & $\%$ or SD & \\
\hline Age (years) & 43.9 & 12.8 & 43.4 & 10.5 & 0.807 & 39.0 & 9.0 & 39.0 & 11.1 & 0.978 \\
\hline \multicolumn{11}{|l|}{ Medical morbidity } \\
\hline - No & 46 & 64.8 & 37 & 58.7 & 0.471 & 75 & 72.1 & 29 & 60.4 & 0.149 \\
\hline - Yes & 25 & 35.2 & 26 & 41.3 & & 29 & 27.9 & 19 & 39.6 & \\
\hline \multicolumn{11}{|l|}{ Smoking } \\
\hline - No & 38 & 53.5 & 35 & 55.6 & 0.813 & 76 & 73.1 & 33 & 68.8 & 0.582 \\
\hline - Yes & 33 & 46.5 & 28 & 44.4 & & 28 & 26.9 & 15 & 31.3 & \\
\hline \multicolumn{11}{|l|}{ Alcohol drinking } \\
\hline - No & 47 & 66.2 & 42 & 66.7 & 0.954 & 83 & 79.8 & 43 & 89.6 & 0.137 \\
\hline - Yes & 24 & 33.8 & 21 & 33.3 & & 21 & 20.2 & 5 & 10.4 & \\
\hline \multicolumn{11}{|l|}{$\mathrm{PA}^{2}$ level } \\
\hline - Low & 39 & 54.9 & 43 & 68.3 & 0.114 & 67 & 64.4 & 34 & 70.8 & 0.437 \\
\hline - Moderate/High & 32 & 45.1 & 20 & 31.7 & & 37 & 35.6 & 14 & 29.2 & \\
\hline \multicolumn{11}{|l|}{ Marital status } \\
\hline $\begin{array}{l}\text { - Single/ Separated/ } \\
\text { Divorced }\end{array}$ & 24 & 33.8 & 21 & 33.3 & 0.954 & 32 & $30.8^{\mathrm{a}}$ & 28 & $58.3^{\mathrm{a}}$ & 0.001 \\
\hline - Married & 47 & 66.2 & 42 & 66.7 & & 72 & $69.2^{\mathrm{a}}$ & 20 & $41.7^{\mathrm{a}}$ & \\
\hline \multicolumn{11}{|l|}{ Education level } \\
\hline - High school & 19 & 26.8 & 18 & 28.6 & 0.124 & 10 & 9.6 & 10 & 20.8 & 0.154 \\
\hline - Bachelor degree & 10 & 14.1 & 17 & 27.0 & & 34 & 32.7 & 15 & 31.3 & \\
\hline - Graduate & 42 & 59.2 & 28 & 44.4 & & 60 & 57.7 & 23 & 47.9 & \\
\hline \multicolumn{11}{|l|}{ Income (\$) } \\
\hline$-<2250$ & 20 & 28.2 & 24 & 38.1 & 0.095 & 25 & 24.6 & 16 & 33.3 & 0.467 \\
\hline$-2250-4000$ & 13 & 18.3 & 17 & 27.0 & & 37 & 35.6 & 14 & 29.2 & \\
\hline$->4000$ & 38 & 53.5 & 22 & 34.9 & & 42 & 40.4 & 18 & 37.5 & \\
\hline \multicolumn{11}{|l|}{$\mathrm{BMI}^{3}\left(\mathrm{Kg} / \mathrm{m}^{2}\right)$} \\
\hline - Underweight & & & & & & 2 & 1.9 & 0 & 0.0 & $<0.001$ \\
\hline - Normal & 21 & $29.6^{\mathrm{a}}$ & 4 & $6.3^{\mathrm{a}}$ & $<0.001$ & 69 & $66.3^{\mathrm{a}}$ & 18 & $37.5^{\mathrm{a}}$ & \\
\hline - Overweight & 37 & 52.1 & 32 & 50.8 & & 25 & 24.0 & 13 & 27.1 & \\
\hline - Obese & 13 & $18.3^{\mathrm{a}}$ & 27 & $42.9^{\mathrm{a}}$ & & 8 & $7.7^{\mathrm{a}}$ & 17 & $35.4^{\mathrm{a}}$ & \\
\hline $\mathrm{PBF}^{4}$ & 25.0 & 6.5 & 29.4 & 6.2 & $<0.001$ & 31.4 & 6.6 & 38.7 & 7.5 & $<0.001$ \\
\hline \multicolumn{11}{|l|}{ Waist circumference risky ${ }^{5}$} \\
\hline- No & 53 & $74.6^{\mathrm{a}}$ & 21 & $33.3^{\mathrm{a}}$ & $<0.001$ & 67 & $64.4^{\mathrm{a}}$ & 11 & $22.9^{\mathrm{a}}$ & $<0.001$ \\
\hline - Yes & 18 & $25.4^{\mathrm{a}}$ & 42 & $66.7^{\mathrm{a}}$ & & 37 & $35.6^{\mathrm{a}}$ & 37 & $77.1^{a}$ & \\
\hline \multicolumn{11}{|c|}{ Sweets or carbonated beverages } \\
\hline$-<1$ beverage/ day & 48 & 67.6 & 38 & 60.3 & 0.380 & 80 & 76.9 & 35 & 72.9 & 0.593 \\
\hline - $\geq 1$ beverage/ day & 23 & 32.4 & 25 & 39.7 & & 24 & 23.1 & 13 & 27.1 & \\
\hline Dairy product intake & 2.1 & 1.1 & 2.3 & 0.9 & 0.344 & 2.2 & 0.9 & 2.1 & 1.1 & 0.534 \\
\hline Glucose (mmol/L) & 5.0 & 0.5 & 5.6 & 1.6 & 0.003 & 4.7 & 0.4 & 5.1 & 0.6 & $<0.001$ \\
\hline Insulin (ulU/ ml) & 8.8 & 1.3 & 16.3 & 8.2 & $<0.001$ & 8.5 & 1.6 & 19.0 & 13.1 & $<0.001$ \\
\hline
\end{tabular}


Table 3 Socio-demographic, lifestyle, anthropometric, and biochemical factors associated with HOMA-IR ${ }^{1}$ (Continued)

\begin{tabular}{|c|c|c|c|c|c|c|c|c|c|c|}
\hline \multirow{4}{*}{ Characteristic } & \multicolumn{10}{|c|}{ HOMA-IR categories } \\
\hline & \multicolumn{5}{|c|}{ Men $(n=134)$} & \multicolumn{5}{|c|}{ Women $(n=152)$} \\
\hline & \multicolumn{2}{|c|}{$\begin{array}{l}\text { HOMA-IR }<2.5 \\
N=71\end{array}$} & \multicolumn{2}{|c|}{$\begin{array}{l}\text { HOMA-IR } \geq 2.5) \\
N=63\end{array}$} & \multirow[b]{2}{*}{$P$-value } & \multicolumn{2}{|c|}{$\begin{array}{l}\text { HOMA-IR }<2.5 \\
N=104\end{array}$} & \multicolumn{2}{|c|}{$\begin{array}{l}\text { HOMA-IR } \geq 2.5) \\
N=48\end{array}$} & \multirow[b]{2}{*}{$P$-value } \\
\hline & $\mathrm{n}$ or mean & $\%$ or SD & $\mathrm{n}$ or mean & $\%$ or SD & & $\mathrm{n}$ or mean & $\%$ or SD & $\mathrm{n}$ or mean & $\%$ or SD & \\
\hline \multicolumn{11}{|l|}{ Hypertriglyceridemia } \\
\hline - Normal TG ${ }^{6}$ levels & 44 & 62.0 & 31 & 49.2 & 0.137 & 99 & $95.2^{\mathrm{a}}$ & 30 & $62.5^{\mathrm{a}}$ & $<0.001$ \\
\hline - Hypertriglyceridemia & 27 & 38.0 & 32 & 50.8 & & 5 & $4.8^{\mathrm{a}}$ & 18 & $37.5^{\mathrm{a}}$ & \\
\hline \multicolumn{11}{|l|}{ Cholesterol $^{7}$} \\
\hline - Desirable & 51 & 71.8 & 39 & 61.9 & 0.222 & 69 & 66.3 & 26 & 54.2 & 0.149 \\
\hline - High & 20 & 28.2 & 24 & 38.1 & & 35 & 33.7 & 22 & 45.8 & \\
\hline \multicolumn{11}{|l|}{$\mathrm{HDL}^{8}$} \\
\hline - Normal & 55 & 77.5 & 45 & 71.4 & 0.423 & 87 & $83.7^{\mathrm{a}}$ & 32 & $66.7^{\mathrm{a}}$ & 0.018 \\
\hline - Low & 16 & 22.5 & 18 & 28.6 & & 17 & $16.3^{\mathrm{a}}$ & 16 & $33.3^{\mathrm{a}}$ & \\
\hline \multicolumn{11}{|l|}{$\operatorname{LDL}^{9}$} \\
\hline - Optimal & 28 & 40.0 & 19 & 30.6 & 0.263 & 39 & 37.5 & 12 & 25.0 & 0.129 \\
\hline - Above optimal & 42 & 60.0 & 43 & 69.4 & & 65 & 62.5 & 36 & 75.0 & \\
\hline \multicolumn{11}{|l|}{$\mathrm{CRP}^{10}$} \\
\hline - Low/ moderate & 26 & 36.6 & 20 & 31.7 & 0.553 & 61 & $58.7^{\mathrm{a}}$ & 17 & $35.4^{\mathrm{a}}$ & 0.008 \\
\hline - High & 45 & 63.4 & 43 & 68.3 & & 43 & $41.3^{\mathrm{a}}$ & 31 & $64.6^{\mathrm{a}}$ & \\
\hline
\end{tabular}

${ }^{1}$ Homeostatic Model Assessment for Insulin Resistance - HOMA-IR levels $\geq 2.5$ indicates insulin resistance [43]

${ }^{2}$ Physical activity

${ }^{3}$ Body Mass Index - BMl $<18.5 \mathrm{~kg} / \mathrm{m}^{2}$, normal weight $18.5-24.9 \mathrm{~kg} / \mathrm{m}^{2}$, overweight $25-29.9 \mathrm{~kg} / \mathrm{m}^{2}$, and obese $\geq 30 \mathrm{~kg} / \mathrm{m}^{2}$ [44]

${ }^{4}$ Percent body fat

${ }^{5}$ Risky waist circumference: $>102 \mathrm{~cm}$ in men, $>88 \mathrm{~cm}$ in women [44]

${ }^{6}$ Triglycerides - Normal levels: $<150 \mathrm{mg} / \mathrm{dL}[45]$

7 Desirable levels: $<200 \mathrm{mg} / \mathrm{dL}[45]$

${ }^{8}$ High Density Lipoprotein - Normal levels: $\geq 40 \mathrm{mg} / \mathrm{dL}, \geq 50 \mathrm{mg} / \mathrm{dL}[45]$

${ }^{9}$ Low Density Lipoprotein - Optimal levels: $<100 \mathrm{mg} / \mathrm{dL}[45]$

${ }^{10}$ C-Reactive Protein - Low/ moderate levels: $<3 \mathrm{mg} / \mathrm{L}[46]$

Columns with superscripts without a common symbol differ, $P<0.05$

Table 4 Logistic regression for dairy product intake and HOMA-IR ${ }^{1}$ after controlling for confounders among males

\begin{tabular}{|c|c|c|c|c|}
\hline \multirow{3}{*}{ Characteristic } & \multicolumn{4}{|c|}{ HOMA-IR } \\
\hline & \multirow{2}{*}{$\begin{array}{l}\text { Odds } \\
\text { Ratio }\end{array}$} & \multicolumn{2}{|c|}{ 95\% Confidence interval } & \multirow[t]{2}{*}{$P$-value } \\
\hline & & Lower & Upper & \\
\hline Age (years) & 0.971 & 0.936 & 1.008 & 0.123 \\
\hline $\mathrm{PA}^{2}$ level & 0.581 & 0.249 & 1.357 & 0.210 \\
\hline$\left.B M\right|^{3}\left(\mathrm{Kg} / \mathrm{m}^{2}\right)$ & 1.378 & 0.590 & 3.220 & 0.458 \\
\hline $\mathrm{PBF}^{4}$ & 1.039 & 0.951 & 1.134 & 0.396 \\
\hline Waist circumference risky ${ }^{5}$ & 3.927 & 1.398 & 11.034 & 0.009 \\
\hline Dairy product intake (serving/ day) & 0.941 & 0.616 & 1.436 & 0.777 \\
\hline Hypertriglyceridemia $^{6}$ & 0.953 & 0.412 & 2.207 & 0.911 \\
\hline
\end{tabular}

${ }_{1}^{1}$ Homeostatic Model Assessment for Insulin Resistance - HOMA-IR levels $\geq 2.5$ indicates insulin resistance [43]

2 Physical activity

${ }^{3}$ Body Mass Index - BMI $<18.5 \mathrm{~kg} / \mathrm{m}^{2}$, normal weight $18.5-24.9 \mathrm{~kg} / \mathrm{m}^{2}$, overweight $25-29.9 \mathrm{~kg} / \mathrm{m}^{2}$, and obese $\geq 30 \mathrm{~kg} / \mathrm{m}^{2}$ [44]

${ }^{4}$ Percent body fat

${ }^{5}$ Risky waist circumference: $>102 \mathrm{~cm}$ in men, $>88 \mathrm{~cm}$ in women [44]

${ }^{6}$ Triglycerides - Normal levels: $<150 \mathrm{mg} / \mathrm{dL}[45]$ 
Table 5 Logistic regression for dairy product intake and HOMA-IR ${ }^{1}$ after controlling for confounders among females

\begin{tabular}{|c|c|c|c|c|}
\hline \multirow{3}{*}{ Characteristic } & \multicolumn{4}{|c|}{ HOMA-IR } \\
\hline & \multirow{2}{*}{$\begin{array}{l}\text { Odds } \\
\text { Ratio }\end{array}$} & \multicolumn{2}{|c|}{ 95\% Confidence interval } & \multirow[t]{2}{*}{$P$-value } \\
\hline & & Lower & Upper & \\
\hline Age (years) & 0.946 & 0.891 & 1.005 & 0.073 \\
\hline Marital status & 0.194 & 0.070 & 0.535 & 0.002 \\
\hline$B M I^{2}\left(K g / m^{2}\right)$ & 1.025 & 0.435 & 2.431 & 0.955 \\
\hline $\mathrm{PBF}^{3}$ & 1.162 & 1.040 & 1.298 & 0.008 \\
\hline Waist circumference risky ${ }^{4}$ & 1.461 & 0.3620 & 5.901 & 0.594 \\
\hline Dairy product intake (serving/ day) & 1.009 & 0.637 & 1.600 & 0.968 \\
\hline Hypertriglyceridemia $^{5}$ & 8.715 & 2.117 & 35.875 & 0.003 \\
\hline $\mathrm{HDL}^{6}$ & 3.056 & 0.951 & 9.823 & 0.061 \\
\hline $\mathrm{CRP}^{7}$ & 0.756 & 0.258 & 2.212 & 0.609 \\
\hline
\end{tabular}

${ }_{1}^{1}$ Homeostatic Model Assessment for Insulin Resistance - HOMA-IR levels $\geq 2.5$ indicates insulin resistance [43]

${ }^{2}$ Body Mass Index - BMl $<18.5 \mathrm{~kg} / \mathrm{m}^{2}$, normal weight $18.5-24.9 \mathrm{~kg} / \mathrm{m}^{2}$, overweight $25-29.9 \mathrm{~kg} / \mathrm{m}^{2}$, and obese $\geq 30 \mathrm{~kg} / \mathrm{m}^{2}$ [44]

${ }^{3}$ Percent body fat

${ }^{4}$ Risky waist circumference: $>102 \mathrm{~cm}$ in men, $>88 \mathrm{~cm}$ in women [44]

${ }^{5}$ Triglycerides - Normal levels: $<150 \mathrm{mg} / \mathrm{dL}$ [45]

${ }^{6}$ High Density Lipoproteins - Normal levels: $\geq 40 \mathrm{mg} / \mathrm{dL}, \geq 50 \mathrm{mg} / \mathrm{dL}$ [45]

${ }^{7}$ C-Reactive Protein - Low/ moderate levels: $<3$ mg/L [46]

inconsistent in the literature. For instance, Ma et al., 2006 and Akter et al., 2013 reported similar results in 1087 US adults with an average age of $54.8 \pm 8.4$ years and 496 Japanese adults aged between 20 and 68 years respectively $[28,31]$, it is important to point out the average or median intake of dairy products reported in all these studies were low. While others [29, 30,32] that found an improvement in insulin resistance upon dairy product consumption, showed higher intakes of dairy products. In contrast, Tucker et al., 2015 showed an increase in insulin resistance among 272 middle aged non-diabetic American women [33]. This disparity in the results obtained could be due to multiple reasons. For instance, some studies were conducted only on one gender [30,33] and on people with a specific BMI range [30, 32, 34] or among people reporting specific conditions (i.e. metabolic syndrome) [32, 34]. In contrast, our sample was largely heterogeneous in terms of gender, BMI and metabolic status. Moreover, results of the bivariate analyses showed that dairy consumption was associated with lower adiposity, which is associated with insulin resistance. However, it could be that the study lacked enough power to detect the protective effect of dairy intake against insulin resistance.

Our study has some limitations that warrant mention. First of all, this study had a cross-sectional design and an association derived from a cross-sectional study does not necessarily indicate causality. Second, the sample size was smaller than the calculated one due to exclusion criteria and missing data. In addition, although our sample is representative of NDU employees, yet it is not representative of the general Lebanese population. Moreover, only $20 \%$ of participants complied with the USDA recommendation for dairy consumption and thus, the described associations are among participants with low to very-low dairy consumption. For the assessment of energy and nutrient intake, 24-h Multiple-Pass Method recall was used. Although this technique has many memory cues that increase the ability of participant to recall food and beverages consumed in the last $24 \mathrm{~h}$, compared to the usual 24-h recall [39], multiple 24 $\mathrm{h}$ recalls could have generated better approximates of usual energy and nutrient intake. On the other hand, the present study has many strengths. To our knowledge, this is the first study in Lebanon and the Middle East to assess the relationship between dairy product intake and insulin resistance. Second, we used two different dietary assessment tools, the FFQ and the 24-h recall MultiplePass Method recall. Moreover, this study controlled for many important confounding variables that were not controlled for in previous studies such as TG, HDL, and CRP levels.

\section{Conclusion}

More research focusing on the relationship between dairy intake and insulin resistance is needed, especially in the Arab and Middle-Eastern region. Further, Middle Eastern dairy products are unique, have different nutrient content and thus might have different effect on insulin resistance. Moreover, several nutrients found in dairy products may have an effect on insulin resistance, whether beneficial or harmful. More studies are needed to elucidate which nutrient has the strongest effect. Finally, future studies should also examine the association between dairy products of different fat content and insulin resistance, as this association might be confounded with the fat content of dairy products. 


\section{Supplementary information}

Supplementary information accompanies this paper at https://doi.org/10. 1186/s12902-020-00558-9.

Additional file 1. Background questionnaire (medical history, sociodemographic and lifestyle questions)

\section{Abbreviations}

ANOVA: One way analysis of variance; BIA: Bioelectrical impedance analysis; BMI: Body mass index; CRP: C - reactive protein; ELISA: Enzyme linked immunosorbent assay; FFQ: Food frequency questionnaire; HDL: High density lipoproteins; HOMA-IR: Homeostatic Model Assessment-Insulin Resistance; LDL: Low density lipoproteins; NDU: Notre Dame University; PA: Physical activity; PBF: Percent body fat; SPSS: Statistical Package for Social Sciences; TG: Triglycerides; US: United States; USDA: United States Department of Agriculture; WHO: World Health Organization

\section{Acknowledgements}

The authors are grateful to the study participants for their cooperation and participation. We also thank the nurses, Ms. Najwa El Gerges, Ms. Micheline Abou Saleh and Ms. Marie Kosseify who assisted in blood withdrawal. And last but not least, we would like to show our gratitude for Notre Dame University, Zouk Mosbeh for giving us access to its laboratories and professional machines, making this research project achievable.

\section{Authors' contributions}

MF, JEHF, SM, JBM and SAH and RG conceived the study and developed the study design and methods; MF, SM, RG, SAH conducted the data collection, entered and analyzed the data; MF, SM interpreted the data. MF, JEHF wrote the paper. MAJ reviewed the paper and provided constructive feedback. All authors read and approved the final version of the manuscript.

\section{Funding}

This research did not receive any funding.

\section{Availability of data and materials}

The datasets used and/or analysed during the current study are available from the corresponding author on reasonable request.

\section{Ethics approval and consent to participate}

Prior to the initiation of the study, the protocol was approved by the Institutional Review Board of Notre Dame University-Louaize. All study participants were informed about the purpose of the study and additional information was given as needed. Written informed consent was obtained from all subjects prior to participation in this study. An identification number was assigned for each participant to protect their identity. All questionnaires were labeled using codes.

\section{Consent for publication}

Not applicable.

\section{Competing interests}

The authors declare that they have no competing interests.

\section{Author details}

${ }^{1}$ Department of Nursing and Health Sciences, Notre Dame UniversityLouaize (NDU), Zouk Mosbeh, Lebanon. ²Department of Endocrinology, Diabetes, Metabolism and Eating Disorders, University Hospital of Saint-Etienne, Saint-Etienne Cedex, France. ${ }^{3}$ Department of Dietetics and Nutrition, The University of Kansas Medical Center, 3901 Rainbow Blvd, Kansas City, KS 66160, USA

Received: 10 January 2020 Accepted: 25 May 2020 Published online: 10 June 2020

\section{References}

1. International Diabetes Federation. IDF Diabetes Atlas. 9th ed; 2019. https://mww.idf. org/.

2. Lebanon. (2016). World Health Organization - Diabetes country profiles. http://www.who.int/diabetes/country-profiles/en/\#L.
3. American Diabetes Association. National Diabetes Statistics Report, 2017: Estimates of diabetes and its burden in the United States. National Center for Chronic Disease Prevention and Health Promotion. http://www.diabetes.org/assets/pdfs/basics/cdcstatistics-report-2017.pdf.

4. American Diabetes Association. Diagnosis and Classification of Diabetes Mellitus. Diabetes Care. 2010;33(Suppl 1):62-9.

5. Tam CS, Xie W, Johnson WT, Cefalu WT, Redman LM, Ravussin E. Defining insulin resistance from Hyperinsulinemic-Euglycemic clamps. Diabetes Care. 2012;35(7): 1605-10.

6. Friedrich $N$, Thuesen $B$, Jørgensen $T$, Juul A, Spielhagen $C$, Wallaschofksi $H$, et al. The association between IGF-I and insulin resistance: a general population study in danish adults. Diabetes Care. 2012;35(4):768-73.

7. Do HD, Lohsoonthorn V, Jiamjarasrangsi W, Lertmaharit S, Williams MA. Prevalence of insulin resistance and its relationship with cardiovascular disease risk factors among thai adults over 35 years old. Diabetes Res Clin Pract. 2010;89(3):303-8.

8. Qu H, Li Q, Rentfro AR, Fisher-Hoch SP, McCormick JB. The definition of insulin resistance using HOMA-IR for americans of mexican descent using machine learning. PLoS One. 2011;6(6):e21041

9. Bermudez V, Salazar J, Martínez MS, Chávez-Castillo M, Olivar LC, Calvo MJ et al. Prevalence and associated factors of insulin resistance in adults from Maracaibo city, Venezuela. Adv Prev Med. 2016;2016:1-13.

10. Naja F, Nasreddine L, Hwalla N, Moghames P, Shoaib H, Fatfat M, et al. Association of $\mathrm{H}$. pylori infection with insulin resistance and metabolic syndrome among lebanese adults. Helicobacter. 2012;17(6):444-51.

11. Gabriely I, Ma XH, Yang XM, Atzmon G, Rajala MW, Berg AH, et al. Removal of visceral fat prevents insulin resistance and glucose intolerance of aging: An adipokine-mediated process? Diabetes. 2002;51(10):2951-8.

12. Petersen KF, Befroy D, Dufour S, Dziura J, Ariyan C, Rothman DL, et al. Mitochondrial dysfunction in the elderly: possible role in insulin resistance. Science. 2003:300(5622):1140-2.

13. Racette SB, Evans EM, Weiss EP, Hagberg JM, Holloszy JO. Abdominal adiposity is a stronger predictor of insulin resistance than fitness among 50-95 year olds. Diabetes Care. 2006;29(3):673-8.

14. Gokulakrishnan K, Deepa M, Monickaraj F, Mohan V. Relationship of body fat with insulin resistance and cardiometabolic risk factors among normal glucose-tolerant subjects. J Postgrad Med. 2011:57(3):184-8.

15. Rytka JM, Wueest S, Schoenle EJ, Konrad D. The portal theory supported by venous drainage-selective fat transplantation. Diabetes. 2011:60(1):56-63.

16. Bonaventura MM, Rodriguez D, Ferreira ML, Crivello M, Repetto EM, Bettler $B$, et al. Sex differences in insulin resistance in GABAB1 knockout mice. Life Sci. 2013:92(3):175-82.

17. Geer EB, Shen W. Gender differences in insulin resistance, body composition, and energy balance. Gender Med. 2009:6(Suppl 1):60-75.

18. Ferrara CM, Goldberg AP, Nicklas BJ, Sorkin JD, Ryan AS. Sex differences in insulin action and body fat distribution in overweight and obese middleaged and older men and women. Appl Physiol Nutr Metab. 2008;33(4):784-90.

19. Hamburg NM, McMackin CJ, Huang AL, Shenouda SM, Widlansky ME, Schulz E, et al. Physical inactivity rapidly induces insulin resistance and microvascular dysfunction in healthy volunteers. Arterioscler Thromb Vasc Biol. 2007:27(12):2650-6.

20. Balkau B, Mhamdi L, Oppert JM, Nolan J, Golay A, Porcellati F, et al. Physical activity and insulin sensitivity: the RISC study. Diabetes. 2008:57(10):2613-8.

21. Mason C, Foster-Schubert KE, Imayama I, Kong A, Xiao L, Bain C, et al. Dietary weight loss and exercise effects on insulin resistance in postmenopausal women. Am J Prev Med. 2011;41(4):366-75.

22. Pan XR, Li GW, Hu YH, Wang JX, Yang WY, An ZX, et al. Effects of diet and exercise in preventing NIDDM in people with impaired glucose tolerance. The da qing IGT and diabetes study. Diabetes Care. 1997;20(4):537-44.

23. Heaney RP. Dairy and bone health. J Am Coll Nutr. 2009;28(Suppl 1):82-90

24. Alqaisi $\mathrm{O}, \mathrm{Ndambi} \mathrm{OA}$, Uddin MM, Hemme T. Current situation and the development of the dairy industry in Jordan, Saudi Arabia, and Syria. Trop Anim Health Prod. 2010;42(6):1063-71.

25. Nasreddine L, Hwalla N, Sibai A, Hamzé M, Parent-Massin D. Food consumption patterns in an adult urban population in Beirut, Lebanon. Public Health Nutr. 2006:9(2):194-203.

26. Farhat $A G$, Jaalouk $D$, Francis $S$. Adherence to the mediterranean diet in a lebanese sample. Nutrition Food Sci. 2016:46(2):272-81.

27. Pan A, Malik V, Hu FB. Exporting diabetes to Asia: the impact of Westernstyle fast food. Circulation. 2012;126(2):163-5.

28. Akter S, Kurotani K, Nanri A, Pham NM, Sato M, Hayabuchi H, et al. Dairy consumption is associated with decreased insulin resistance among the japanese. Nutr Res (New York, N.Y.). 2013;33(4):286-92. 
29. Drehmer M, Pereira MA, Schmidt MI, Del Carmen Molina BM, Alvim S, Lotufo PA, et al. Associations of dairy intake with glycemia and insulinemia, independent of obesity, in brazilian adults: the brazilian longitudinal study of adult health (ELSA-brasil). Am J Clin Nutr. 2015;101(4):775-82.

30. Hashemipour S, Esmailzadehha N, Mohammadzadeh M, Ziaee A. Association of meat and dairy consumption with normal weight metabolic obesity in men: the Qazvin metabolic diseases study. Eat Weight Disord. 2016;21(3): 419-25.

31. Ma B, Lawson AB, Liese AD, Bell RA, Mayer-Davis EJ. Dairy, magnesium, and calcium intake in relation to insulin sensitivity: approaches to modeling a dose-dependent association. Am J Epidemiol. 2006;164(5):449-58.

32. Stancliffe R, Thorpe T, Zemel M. Dairy attentuates oxidative and inflammatory stress in metabolic syndrome. Am J Clin Nutr. 2011;94(2):422-30.

33. Tucker LA, Erickson A, LeCheminant JD, Bailey BW. Dairy consumption and insulin resistance: the role of body fat, physical activity, and energy intake. J Diab Res. 2015;2015:1-11.

34. Wennersberg MH, Smedman A, Turpeinen AM, Retterstøl K, Tengblad S,

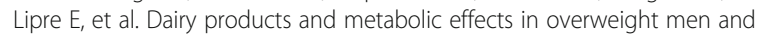
women: results from a 6-mo intervention study. Am J Clin Nutr. 2009;90(4): 960-8.

35. Ramachandran A, Snehalatha C, Shetty AS, Nanditha A. Trends in prevalence of diabetes in Asian countries. World J Diabetes. 2012;3(6):110-7.

36. Al Hayek S, Matar Bou Mosleh J, Ghadieh R, El Hayek Fares J. Vitamin D status and body composition: a cross-sectional study among employees at a private university in Lebanon. BMC Nutr. 2018;4:31.

37. Booth M. Assessment of physical activity: An international perspective. Res Q Exerc Sport. 2000;71(2):114-20.

38. El Hayek J, Pham T, Finch S, Hazell T, Vanstone C, Weiler H. Validity and reproducibility of a short food frequency questionnaire in assessing calcium and vitamin D intake in Canadian preschoolers. EC Nutrition. 2014;1(1):9-18.

39. Steinfeldt L, Anand J, Murayi T. Food reporting patterns in the USDA automated multiple-pass method. Proceda Food Sci. 2013;2:145-56.

40. Matthews DR, Hosker JP, Rudenski AS, Naylor BA, Treacher DF, Turner RC. Homeostasis model assessment: insulin resistance and beta cell function from fasting plasma glucose and insulin concentrations in man. Diabetologia. 1985;28(4):412-9.

41. Bonora E, Targher G, Alberiche M, Bonadonna RC, Saggiani F, Zenere MB, et al. Homeostasis model assessment closely mirrors the glucose clamp technique in the assessment of insulin sensitivity: studies in subjects with various degrees of glucose tolerance and insulin sensitivity. Diabetes Care. 2000;23(1):57-63.

42. Antuna-Puente B, Disse E, Rabasa-Lhoret R, Laville M, Capeau J, Bastard J. How can we measure insulin sensitivity/resistance? Diabetes Metab. 2011; 37(3):179-88.

43. Gutch M, Kumar S, Razi SM, Gupta KK, Gupta A. Assessment of insulin sensitivity/resistance. Indian J Endocrinol Metab. 2015;19(1):160-4.

44. WHO. Waist circumference and waist-hip ratio. Report of a WHO Expert Consultation. Geneva: World Health Organization; 2008. p. 8-11.

45. National Institutes of Health: Third Report of the National Cholesterol Education Program Expert Panel on Detection, Evaluation, and Treatment of High Blood Cholesterol in Adults (Adult Treatment Panel III): Executive Summary. Bethesda, MD, National Institutes of Health, 2001 (NIH publ. no. 01-3670).

46. Salazar J, Martínez MS, Chávez M, Toledo A, Añez R, Torres Y, et al. Creactive protein: clinical and epidemiological perspectives. Cardiol Res Pract. 2014:1-10.

47. ChooseMyPlate. All About The Dairy Group. United States Department of Agriculture. https://www.choosemyplate.gov/eathealthy/dairy.

48. Al-Rubeaan K. Type 2 diabetes mellitus red zone; 2010. https://doi.org/10. 1016/j.ijdm.2009.12.009

49. Cnop M, Havel PJ, Utzschneider KM, Carr DB, Sinha MK, Boyko EJ, et al. Relationship of adiponectin to body fat distribution, insulin sensitivity and plasma lipoproteins: evidence for independent roles of age and sex. Diabetologia. 2003;46(4):459-69.

50. The Food-Based Dietary Guideline Manual for Promoting Healthy Eating in the Lebanese Adult Population. The Faculty of Agricultural and Food Sciences, American University of Beirut, Beirut, Lebanon; 2013.

51. Dehghan M, Al Hamad N, Yusufali A, Nusrath F, Yusuf S, Merchant AT. Development of a semi-quantitative food frequency questionnaire for use in United Arab Emirates and Kuwait based on local foods. Nutr J. 2005;4(18). https://doi.org/10.1186/1475-2891-4-18.
52. Esmaillzadeh A, Azadbakht L. Dairy consumption and circulating levels of inflammatory markers among Iranian women. Public Health Nutr. 2010;13(9): 1395-402.

53. Mirmiran P, Esmaillzadeh A, Azizi F. Dairy consumption and body mass index: An inverse relationship. Int J Obes. 2005;29(1):115-21.

54. Shin S, Lee HW, Kim CE, Lim J, Lee J, Kang D. Association between Milk consumption and metabolic syndrome among Korean adults: results from the health examinees study. Nutrients. 2017;9(10):e1102. https://doi.org/10. 3390/nu9101102.

55. Lee KW, Cho W. The consumption of dairy products is associated with reduced risks of obesity and metabolic syndrome in Korean women but not in men. Nutrients. 2017;9(6):630.

56. Merino J, Mateo-Gallego R, Plana N, Bea AM, Ascaso J, Lahoz C, et al. Lowfat dairy products consumption is associated with lower triglyceride concentrations in a Spanish hypertriglyceridemic cohort. Nutricion Hospitalaria. 2013;28(3):927-33.

57. Bendsen NT, Hother A, Jensen SK, Lorenzen JK, Astrup A. Effect of dairy calcium on fecal fat excretion: a randomized crossover trial. Int J Obes. 2008; 32(12):1816-24.

58. Buchowski MS, Aslam M, Dossett C, Dorminy C, Choi L, Acra S. Effect of dairy and non-dairy calcium on fecal fat excretion in lactose digester and maldigester obese adults. Int J Obes. 2010;34(1):127-35.

59. Park SY, Murphy SP, Wilkens LR, Stram DO, Henderson BE, Kolonel LN. Calcium, vitamin $\mathrm{D}$, and dairy product intake and prostate cancer risk: the multiethnic cohort study. Am J Epidemiol. 2007;166(11):1259-69.

60. Lee HJ, Ji C, HSH L, Ci K, Cho E. Intakes of Dairy Products and Calcium and Obesity in Korean Adults: Korean National Health and Nutrition Examination Surveys (KNHANES) 2007-2009. PLoS ONE. 2014;9(6):e99085.

61. Fleming KH, Heimbach JT. Consumption of calcium in the U.S.: food sources and intake levels. J Nutr. 1994;124(Suppl 8):1426-30.

62. McCarron D, Morris C, Henry H, Stanton J. Blood pressure and nutrient intake in the United States. Science. 1984;224(4656):1392-8.

63. Zemel MB, Shi H, Greer B, Dirienzo D, Zemel PC. Regulation of adiposity by dietary calcium. FASEB J. 2000;14(9):1132-8.

64. Lorenzen JK, Nielsen S, Holst JJ, Tetens I, Rehfeld JF, Astrup A. Effect of dairy calcium or supplementary calcium intake on postprandial fat metabolism, appetite, and subsequent energy intake. Am J Clin Nutr. 2007;85(3):678-87.

65. Pfeuffer M, Schrezenmeir J. Milk and the metabolic syndrome. Obes Rev. 2007:8(2):109-18.

66. Chung JO, Cho DH, Chung DJ, Chung MY. Associations among body mass index, insulin resistance, and pancreatic ß-cell function in korean patients with new-onset type 2 diabetes. Korean J Intern Med. 2012;27(1):66-71.

67. Keska A, Lutoslawska G, Czajkowska A, Tkaczyk J, Mazurek K. Variability in HOMA-IR, lipoprotein profile and selected hormones in young active men. TheScientificWorldJournal. 2013. https://doi.org/10.1155/2013/412764.

\section{Publisher's Note}

Springer Nature remains neutral with regard to jurisdictional claims in published maps and institutional affiliations.

\section{Ready to submit your research? Choose BMC and benefit from:}

- fast, convenient online submission

- thorough peer review by experienced researchers in your field

- rapid publication on acceptance

- support for research data, including large and complex data types

- gold Open Access which fosters wider collaboration and increased citations

- maximum visibility for your research: over $100 \mathrm{M}$ website views per year

At $\mathrm{BMC}$, research is always in progress.

Learn more biomedcentral.com/submissions 